\title{
Design of handhold terminal of equipment management system based on Windows CE
}

\author{
Xiaojiao Tang \\ Tangshan Key Laboratory of Mechantronics, Tangshan College, Hebei Tangshan, China 063000
}

Keywords: Handhold terminal ; equipment management ; cloud culculating ;Windows CE; RDA

\begin{abstract}
Equipment Management level decides the production efficiency and the cost of production. Traditional equipment management has many problems, such as lower efficiency, decentralized information and so on. According to the demand of coal enterprise and the modern development requirements of coal mine electromechanical device management and repair system, the application system of equipment management, especially the handhold terminal, was developed based Windows CE. Human-computer interaction interface for each function module was developed. The handhold terminal can realize wireless connection and synchronization technology for instrument and equipment management system. It can realize equipment spot-inspection work effectively, improve the reliability of equipment and reduce the incidence of failure.
\end{abstract}

\section{Introduction}

The level of equipment management and maintenance decides an enterprise's production efficiency and production safety. Coalmine equipments have some characteristics, such as complex production environment, equipment multiplicity, wide distribution, enormous information, expensive price, and so on. So it is necessary for coalmine enterprises to improve equipments reliability, decrease incidence rates and reduce maintenance cost. The equipment management system based on cloud calculating can meet the need of coalmine enterprises.

\section{Structure of equipment management system}

The structure of equipment management system is shown in Fig.1. Wireless sensor network nodes are installed on equipments. Handhold terminals collect data of all kinds of equipments and then upload data to enterprise server by wireless modules. The Massive Data are sent into Cloud server. Between handhold terminal and enterprise server, data can be exchanged through USB or WIFI. Computer name must be input into the Dialog box of interface server when using USB and server IP address and port number should be input when using WIFI. The communication of handhold terminal and server is finished simultaneously by RDA (Remote Data Access). It can perform data synchronous communication with the server by wireless network, which includes data up-download and update.

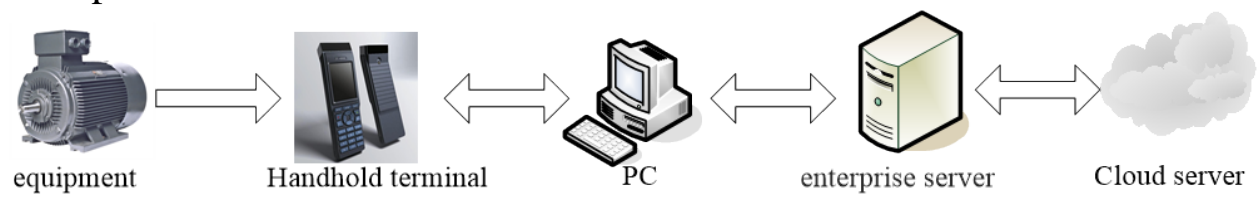

Fig. 1 structure of the system

\section{Design of handhold terminal system}

Handhold terminal system was developed on the platform of Windows CE 6.0 operating systems. Embedded database SQLCE 3.5 was used as its internal database and PowerDesigner15 was used to realize database modeling and build data sheets.

Function modules of handhold terminal consists of six modules, i.e. login module, RFID Regional card module, spot checking module, communication module and auxiliary functions 
module. Login module is divided into account password module and RFID recognition module. After successful authentication, the technician can download spot checking task data and fault data. RFID Regional card module reads RFID card numbers and matches with spot checking data. After successful matching, the system begins to collect data. Spot checking module query fault sheets for those abnormal data and began maintenance work according standard operation. Communication module download operation standard data and upload collected spot checking data. Auxiliary functions module can collect vibration and temperature data.

Removable embedded database SQL CE is used in the handhold terminal, which is mainly used in the removable equipments to manage data. It has powerful Search and query capabilities. The system's removable database consists of four parts: basic information data sheet, spot checking task data sheet, fault data sheet and maintenance standard date sheet.

Basic information data sheet is used to record staff information, equipment categories information and equipment account information. All the relationships between sheets is shown in Fig.2.

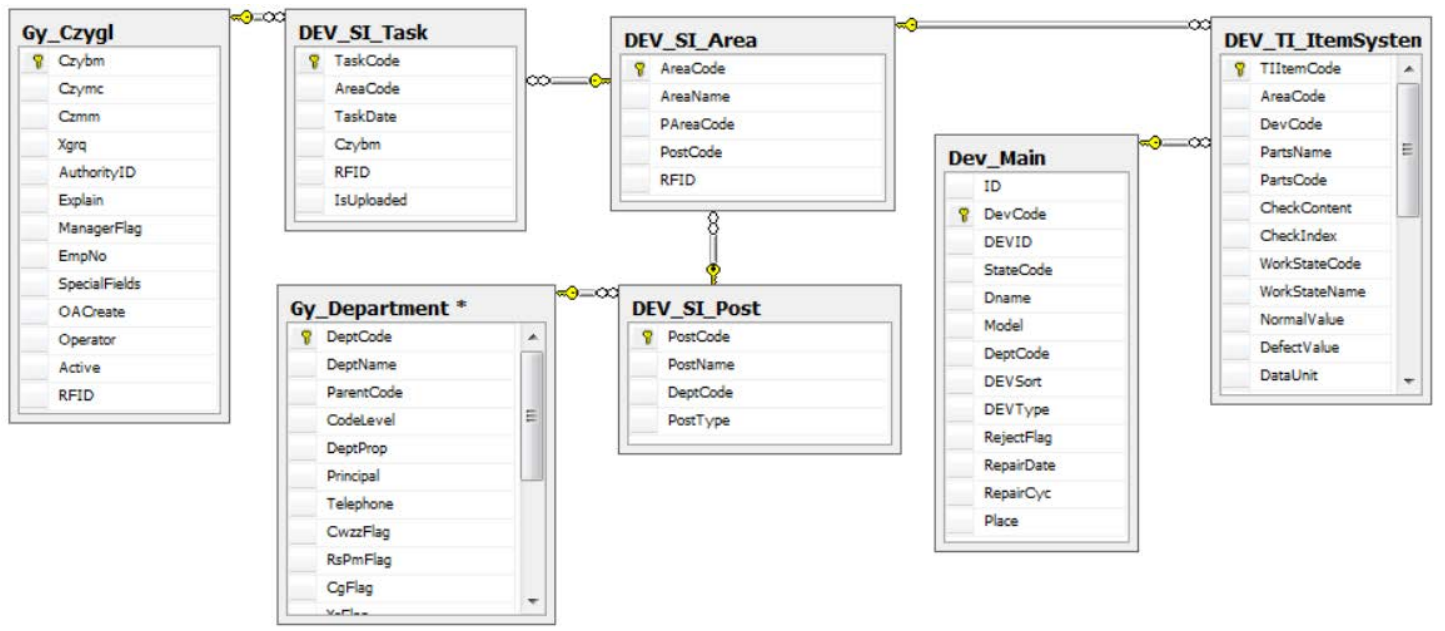

Fig.2 relationships between basic information sheets

Spot checking task data sheet is used to manage spot checking task. According to the inspection task process, it builds inspection postcode sheet, inspection area sheet, inspection operation standard sheet, checked items sheet and so on.

Fault data sheet includes fault cause sheet and fault system sheet. It is used to manage the information of fault cause and fault time. Fault cause sheet is connected with inspection operation standard sheet according fault code. The relationships between the sheets is shown in Fig.3

\begin{tabular}{|c|c|c|c|c|c|c|}
\hline \multicolumn{2}{|c|}{ DEV_SI_FaultSysten } & & \multicolumn{2}{|c|}{ DEV_SI_Fault_Cause } & & DEV_SI_OverhaulSystem \\
\hline 8 & FaultCode & & 8 & FaultCauseCode & & B OverhaulCode \\
\hline & FaultName & & & FaultCauseName & & OverhaulName \\
\hline & DevTypeCode & & & FaultCode & & DevTypeCode \\
\hline & PartsName & $\infty \cdots \infty$ & & OverhaulCode & & IsWorkOrder \\
\hline & PartsCode & & & OverhaulName & $\infty x=c$ & CreateDate \\
\hline & CreateDate & & & CreateDate & & Creator \\
\hline & Creator & & & Creator & & ModifyDate \\
\hline & ModifyDate & & & ModifyDate & & Modifier \\
\hline & Modifier & & & Modifier & & IsValid \\
\hline
\end{tabular}

Fig.3 relationships between fault data sheets

Maintenance standard date sheet is used to manage maintenance process aiming to fault cause. It includes personnel, material, equipment, process, safety measures of maintenance, and so on.

The inspection system synchronizes data with RDA (Remote Data Access) mode. It's realized as follows:

SqlCeRemoteDataAccess rda = new SqlCeRemoteDataAccess();

rda.LocalConnectionString = Settings.AppSettings.ConnectionString; 


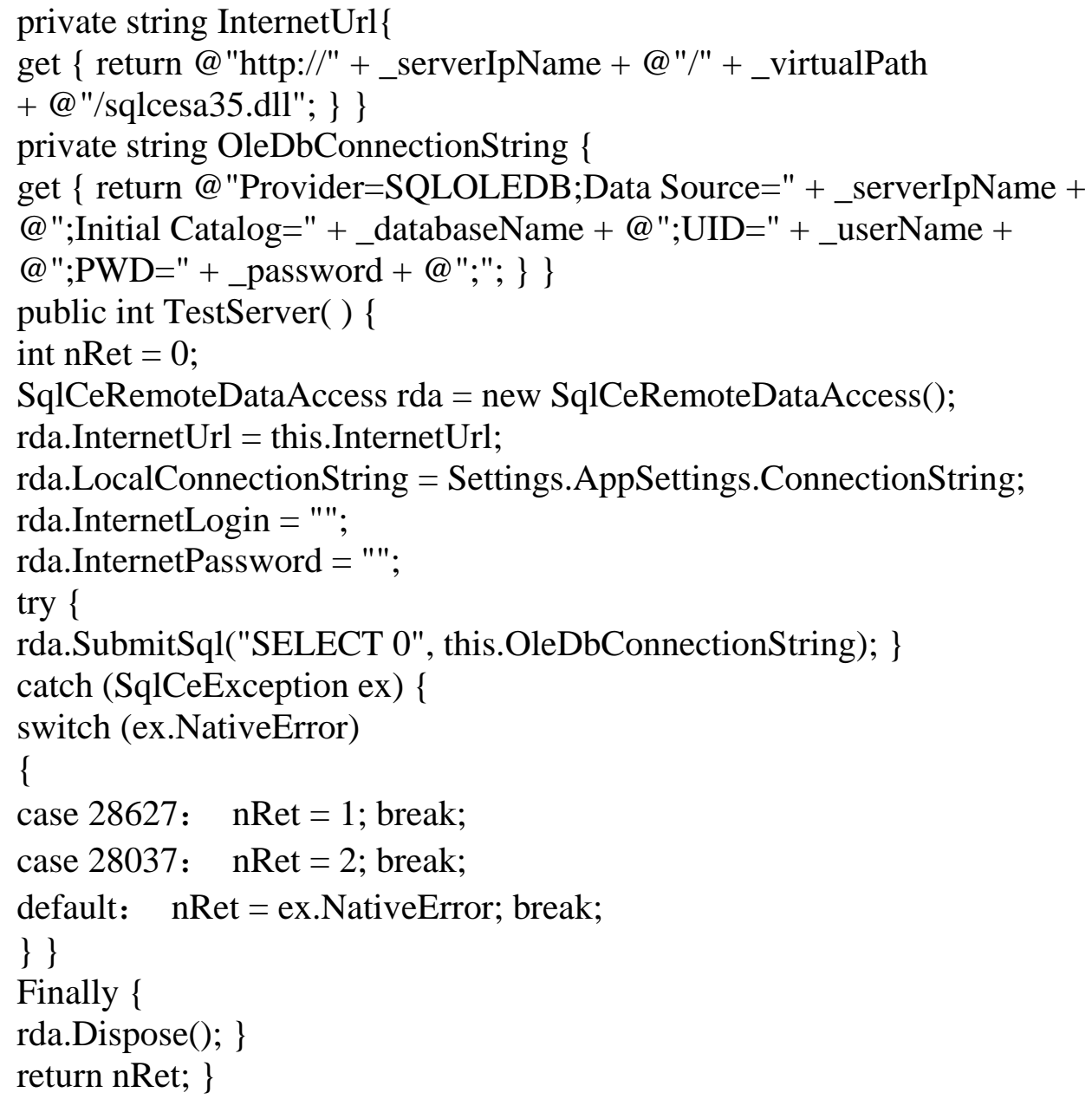

\section{Conclusion}

In this paper, the handhold terminal system was introduced, which was developed on the platform of Windows CE 6.0. Embedded database SQLCE 3.5 was used as its internal database and PowerDesigner15 was used to realize database modeling and build data sheets. Function modules of handhold terminal consists of six modules, i.e. login module, RFID Regional card module, spot checking module, communication module and auxiliary functions module. Login module is divided into account password module and RFID recognition module. Handhold terminal and enterprise server can exchange data through WIFI or USB. This system has been applied in coal enterprises. It operates steadily and is able to effectively implement the measurement and management of equipment spot checking, improving the reliability of equipment and reducing the failure rate of equipment.

\section{References}

[1] Petriu E M, Geoganas N D,Petriu D C et al. Sensor-based information appliances. IEEE Instrumentation and Measurement Magazine. 2000(10):31-35

[2] Song Gui-rong, Cao Hong. Design of Mining Machinery Fault Diagnosis Integration Platform. Coal Mine Machinery. 2013.9,Vol.34 No.09

[3] An-Hsiang Wang. Effects of Palm and WinCE menu-design for PDA on users operating performance and subjective preference .Displays, 2005, 97 (102) :97-102.

[4] Tan Zhang-lu, Zhang Chang-lu, YU Jin-zhi. Research on Construction of Equipment Management System in Coal Mines Based on Application of IOT. Coal Mine Machinery.2013.6, Vol.34 No.06 
[5] Mei Hong,Zhang Lu,Yang Fuqing. A component-based software configuration management model and its supporting system.Journal of Computer Science and Technology.2002,17(4): 432-441. 